\title{
Resultados da cirurgia do aneurisma da aorta abdominal em pacientes jovens
}

\author{
Outcomes after surgical repair of abdominal aortic aneurysms in young \\ patients
}

Telmo P. Bonamigo ${ }^{1}$, Márcio Luís Lucas ${ }^{2}$, Lígia Caon Pereira ${ }^{3}$

\section{Resumo}

Contexto: A presença de aneurisma da aorta abdominal (AAA) é rara em pacientes jovens.

Objetivo: Avaliar os resultados da cirurgia do AAA em pacientes com idade $\leq 50$ anos.

Métodos: De junho de 1979 a janeiro de 2008, 946 pacientes foram operados eletivamente do AAA infrarrenal pelo primeiro autor. Desses, 13 pacientes $(1,4 \%)$ tinham idade $\leq 50$ anos no momento da cirurgia. Foram analisadas as características demográficas e cirúrgicas bem como os resultados precoces e tardios da cirurgia nesses pacientes.

Resultados: A média de idade foi $46 \pm 3,4$ anos (variando de 43 a 50 anos), sendo a maioria homens ( $76,9 \%)$, hipertensos $(76,9 \%)$ e tabagistas (61,5\%). Não ocorreu nenhum óbito perioperatório, e a morbidade cirúrgica atingiu dois pacientes $(15,4 \%)$ (um paciente com infecção respiratória e outro com angina instável). O seguimento mediano foi de 85,5 meses, ocorrendo dois óbitos devido a cardiopatia isquêmica em um paciente e acidente vascular cerebral em outro, durante o seguimento.

Conclusão: A cirurgia do AAA é um procedimento seguro com bons resultados a longo prazo, pois, em nosso estudo, apresentou mortalidade perioperatória nula e boa sobrevida a longo prazo.

Palavras-chave: Aneurisma da aorta abdominal, pessoas jovens, cirurgia.

\section{Introdução}

A prevalência do aneurisma da aorta abdominal (AAA) aumenta com a idade, com uma ocorrência maior em pacientes da sétima e oitava décadas ${ }^{1,2}$. Existem poucos estudos acerca dos resultados após a cirurgia vascular em pacientes jovens, sobretudo nos portadores de $\mathrm{AAA}^{3,4}$.

Nosso objetivo foi avaliar os resultados da cirurgia do AAA degenerativo (aterosclerótico) em pacientes com

\begin{abstract}
Background: Abdominal aortic aneurysms (AAA) are rare in young patients.

Objective: To evaluate outcomes after AAA repair in patients aged $\leq 50$ years.

Methods: Between June 1979 and January 2008, 946 patients underwent elective repair for an infrarenal AAA performed by the first author. Of these, 13 patients $(1.4 \%)$ were $\leq 50$ years old at surgery. Demographic characteristics and surgical data were analyzed, as well as
\end{abstract} early and late outcomes after surgical intervention.

Results: Mean age was $46 \pm 3.4$ years (ranging from 43 to 50 years). Most patients were men (76.9\%), hypertensive $(76.9 \%)$ and smokers $(61.5 \%)$. Perioperative morbidity and mortality rates were low $(15.4 \%$ and $0 \%$, respectively); one patient had respiratory infection and another patient had unstable angina. Median follow-up was 85.5 months, and two patients died due to ischemic cardiopathy and cerebrovascular accident during the follow-up period.

Conclusion: AAA repair in young patients is a safe procedure, with good long-term results. In our study, there were no perioperative deaths, and a good long-term survival was observed.

Keywords: Abdominal aortic aneurysm, young people, surgery.

idade $\leq 50$ anos, comparando-os com aqueles obtidos por outros autores na literatura.

\section{Métodos}

Revisamos os prontuários e protocolos desenvolvidos pelo primeiro autor de pacientes operados eletivamente de AAA infrarrenal entre junho de 1979 a janeiro de 2008. Foram separados os dados de pacientes com idade $\leq 50$

1. Professor titular, Cirurgia Vascular, Universidade Federal de Ciências da Saúde de Porto Alegre (UFCSPA), Porto Alegre, RS. Chefe, Serviço de Cirurgia Vascular, Irmandade Santa Casa de Misericórdia de Porto Alegre (ISCMPA), Porto Alegre, RS.

2. Cirurgião vascular, ISCMPA, Porto Alegre, RS.

3. Cirurgiã vascular, ISCMPA, Porto Alegre, RS.

Não foram declarados conflitos de interesse associados à publicação deste artigo.

Artigo submetido em 27.04.08, aceito em 30.03.09.

J Vasc Bras. 2009;8(2):139-142.

Copyright $@ 2009$ by Sociedade Brasileira de Angiologia e de Cirurgia Vascular 
anos, portadores de AAA degenerativo, submetidos à cirurgia no mesmo intervalo de tempo. Os pacientes com aneurismas congênitos, pseudoaneurismas, arterites, aneurismas suprarrenais ou aneurismas micóticos foram excluídos do estudo.

Obtivemos os dados demográficos e cirúrgicos através dos prontuários e protocolos médicos. O seguimento dos pacientes foi feito através de consultas médicas, entrevista telefônica ou questionário enviado por via postal com o pedido ao médico clínico do paciente para preencher os dados do questionário. Tal conduta permitiu o acompanhamento a longo prazo e identificação das causas de óbitos tardios dos pacientes.

Também foram relatados os dados da mortalidade e morbidade perioperatórias. As complicações perioperatórias foram descritas conforme Copeland et al. ${ }^{5}$ : hemorragia (ferida operatória ou intra-abdominal), infecção (ferida operatória, respiratória, urinária, septicemia), deiscência de ferida operatória, tromboembolismo pulmonar, trombose venosa profunda dos membros inferiores, acidente vascular cerebral, infarto agudo do miocárdio, insuficiência ventilatória, insuficiência cardíaca, falência renal, trombose do enxerto vascular e colite isquêmica.

Os dados obtidos foram expressos em valores absolutos e relativos (porcentagens), sendo calculada a média e o desvio padrão dos valores quando necessário.

\section{Resultados}

Foram submetidos 946 pacientes à cirurgia eletiva do AAA infrarrenal entre junho de 1979 e janeiro de 2008. Identificamos 13 pacientes $(1,4 \%)$ com idade $\leq 50$ anos, sendo a média de idade de 46 $\pm 3,4$ anos (variando de 43 a 50 anos). Dez pacientes eram do sexo masculino (76,9\%), 10 portadores de hipertensão arterial (76,9\%) e 8 com história de tabagismo $(61,5 \%)$ (Tabela 1).

A maioria dos pacientes $(69,2 \%)$ não apresentava sintomas relacionados ao AAA no momento da cirurgia. Dos pacientes sintomáticos $(30,8 \%)$, dois apresentavam dor lombar e dois apresentavam dor abdominal difusa, porém sem diagnóstico de ruptura do AAA. O diâmetro maior médio dos aneurismas foi de $5,6 \pm 1,5 \mathrm{~cm}$ (variando de 3,5 a $9 \mathrm{~cm}$ ), havendo envolvimento das artérias ilíacas em 2 ca$\operatorname{sos}(15,4 \%)$ e presença de aneurisma inflamatório em outros 2 casos $(15,4 \%)$.
Tabela 1 - Comorbidades e dados demográficos de pacientes com idade $\leq 50$ anos submetidos à cirurgia do aneurisma de aorta abdominal

\begin{tabular}{lc}
\hline Comorbidades & $\mathrm{n}(\%)$ \\
\hline Proporção H/F & $10 / 3$ \\
Média de idade & $46 \pm 3,4$ anos \\
Hipertensão arterial & $10(76,9)$ \\
Tabagismo & $8(61,5)$ \\
Cardiopatia isquêmica* & $3(23,1)$ \\
DPOC & $2(15,4)$ \\
Disfunção renal prévia ${ }^{\dagger}$ & $1(7,7)$ \\
Diabetes melito & $0(0)$ \\
\hline
\end{tabular}

DPOC $=$ doença pulmonar obstrutiva crônica; $\mathrm{H} / \mathrm{F}=$ homens $/ \mathrm{mu}$ lheres.

* Presença de angina estável, história de infarto agudo do miocárdio e/ou revascularização do miocárdio prévia.

${ }^{\dagger}$ Níveis de creatinina sérica $=2,0 \mathrm{mg} / \mathrm{dL}$.

Entre os exames complementares, foi realizada radiografia simples do abdômen $(n=3)$, principalmente nos pacientes da fase incial do estudo; eco-Doppler colorido $(\mathrm{n}=5)$; aortografia $(\mathrm{n}=3)$; e tomografia computadorizada de abdômen $(n=4)$.

Todos os pacientes receberam anestesia geral, com abordagem da aorta via transperitoneal, na maioria das vezes por incisão xifo-pubiana $(\mathrm{n}=12)$, sendo realizado acesso retroperitoneal em um paciente. O tempo cirúrgico médio foi de 183,2 $\pm 49,2$ minutos (120-270 minutos), com tempo médio de pinçamento aórtico de $42,8 \pm 16,7$ minutos (23-75 minutos). A reconstrução aórtica compreendeu anastomose aorto-aórtica $(\mathrm{n}=5)$; aorto-biilíaca $(\mathrm{n}=5)$; aorto-ilíaco esquerdo e aorto-femoral direito $(\mathrm{n}=1)$; e aorto-bifemoral $(\mathrm{n}=2)$.

Não ocorreu nenhum óbito perioperatório. Houve complicações em dois pacientes $(15,4 \%)$, sendo infecção respiratória em um deles e angina instável em outro. A média dos níveis de creatinina pré-operatória $(1,34 \pm 0,3 \mathrm{mg} / \mathrm{dL})$ não diferiu estatisticamente dos níveis no pós-operatório $(1,0 \pm 0,08 \mathrm{mg} / \mathrm{dL})(\mathrm{p}>0,05)$.

O seguimento mediano foi de 85,5 meses (variação de 10 a 232 meses). Os dados do seguimento estavam disponíveis em 12 dos 13 pacientes, havendo perda dos dados em somente um paciente $(7,7 \%)$. Ocorreram dois óbitos durante o seguimento, sendo secundários a infarto agudo do miocárdio em um paciente após 64 meses da cirurgia, e 
a acidente vascular cerebral em outro paciente após 60 meses da cirurgia (Tabela 2).

\section{Discussão}

A doença aterosclerótica precoce pode afetar pacientes com idade $\leq 50$ anos. Sua manifestação pode ser doença vascular periférica, cardiopatia isquêmica e doença cerebrovascular $^{6-8}$. A ocorrência de AAA degenerativo em pacientes jovens é rara e existem poucos estudos que relatam os resultados do tratamento cirúrgico em tais pacientes $^{3,4}$.

Neste estudo, relatamos o resultado da cirurgia do AAA em uma série de 13 pacientes jovens, em um total de 946 pacientes operados no período em estudo, perfazendo uma proporção de 1,4\%, semelhante à proporção de 1,6\% encontrada por Cherr et al. ${ }^{4}$. Em nosso estudo, englobamos apenas pacientes com AAA degenerativo infrarrenal. No estudo de Muluk et al. ${ }^{3}$, em que foram relatados 26 casos, a maioria $(n=20)$ era portador de aneurismas desse tipo.

Acredita-se que os pacientes jovens apresentam aneurismas maiores e mais extensos com sintomas mais frequentes do que os pacientes mais velhos. No entanto, no trabalho de Cherr et al. ${ }^{4}$ não foi demonstrada nenhuma diferença nesses parâmetros entre pacientes jovens e mais velhos. Já no estudo de Muluk et al. ${ }^{3}$, a proporção de pacientes com sintomas no momento da cirurgia foi de $46 \%$ no grupo de pacientes jovens e de $6,7 \%$ no grupo controle. Ainda nesse mesmo estudo, foi constatado que o envolvimento aneurismático da aorta proximal (suprarrenal) foi três vezes mais comum nos pacientes jovens ${ }^{3}$. Embora não sendo um estudo comparativo, observamos uma frequência de sintomas nos pacientes jovens de 30,8\%.

Aparentemente, os fatores de risco para doenças cardiovasculares mais prevalentes nos pacientes jovens (hipertensão arterial e tabagismo) são os mesmos relatados

Tabela 2 - Resultados cirúrgicos em pacientes com idade $\leq 50$ anos submetidos à cirurgia do aneurisma de aorta abdominal

\begin{tabular}{ll}
\hline Morbidade, n $(\%)$ & $2(15,4)$ \\
Causas & $\begin{array}{l}\text { - Angina instável } \\
\text { - Infecção respiratória }\end{array}$ \\
Mortalidade, n (\%) & 0 \\
Seguimento mediano & 85,5 meses \\
$\begin{array}{ll}\text { Causas de óbitos tardios } \\
\text { (n) }\end{array}$ & - Infarto agudo do miocárdio $(\mathrm{n}=1)$ \\
\hline
\end{tabular}

para pacientes mais velhos. No estudo de Cherr et al. ${ }^{4}$, não houve diferença estatística significativa dos fatores de risco (cardiopatia isquêmica, hipertensão arterial, tabagismo, diabetes melito, insuficiência renal prévia) entre os pacientes jovens e os mais velhos. No trabalho de Mingoli et al. ${ }^{6}$, sobre o tratamento da doença oclusiva aorto-ilíaca em pacientes jovens, foi demonstrado uma frequência maior de tabagismo, hipertensão e dislipidemia nos pacientes jovens em comparação aos mais velhos.

Em nosso estudo não houve nenhum óbito, e as complicações ocorreram em dois pacientes $(15,4 \%)$. O estudo de Cherr et al., de 19 pacientes, englobou três pacientes com AAA roto (16\%) e dois pacientes com aneurisma suprarrenal (11\%). O índice de mortalidade nesse estudo foi de $16 \%$, não se identificando diferença estatística do grupo controle $(9 \%)^{4}$. A taxa de complicações nesse mesmo estudo foi de $41 \%$ nos pacientes jovens ( $21 \%$ de complicações maiores) e de $35 \%$ no grupo controle ( $9 \%$ de complicações maiores). Em nosso estudo, a taxa de complicações foi de $15,4 \%$.

Devido à boa expectativa de vida nos pacientes mais jovens, é necessário obtermos um bom resultado a longo prazo após a cirurgia. Em nosso estudo, apenas dois pacientes morreram durante um seguimento mediano de 85,5 meses, sendo cardiopatia isquêmica e doença cerebrovascular as duas causas de óbitos. No estudo de Cherr et al. ${ }^{4}$, a sobrevida dos pacientes jovens operados em 3 anos foi de $73 \%$, comparável ao grupo mais velho (69\%). Conforme nossos resultados, as manifestações de doença cardiovascular também foram as principais causas de óbito tardio nos pacientes jovens como relatados por outros autores ${ }^{4,7}$. Em estudo prévio publicado por nós, também observamos que a doença cardíaca e cerebrovascular são as principais causas de óbito tardio nos pacientes mais velhos submetidos à cirurgia do $\mathrm{AAA}^{9}$.

Com relação aos resultados do tratamento cirúrgico de pacientes jovens com outras doenças vasculares, foi demonstrado por Mingoli et al. ${ }^{6}$ e Harris et al. ${ }^{10}$ semelhança dos resultados com relação à perviedade da derivação e sobrevida a longo prazo de pacientes jovens em comparação a pacientes mais velhos submetidos ao tratamento cirúrgico da doença oclusiva aorto-ilíaca e infrainguinal, respectivamente. Na doença carotídea, embora os pacientes mais jovens tenham apresentado maior prevalência de sintomas pré-operatórios, não ficou demonstrada diferença na so- 
brevida e na taxa de re-estenose entre jovens e velhos após a endarterectomia carotídea no estudo de Martin et al. ${ }^{8}$.

Concluindo, a ocorrência de AAA em pacientes jovens é rara. Em nosso estudo, o tratamento cirúrgico nesses pacientes demonstrou uma mortalidade perioperatória nula com taxa de complicação aceitável e boa sobrevida a longo prazo. As manifestaões da doença cardíaca isquêmica e da doença cerebrovascular foram as causas de óbito tardio em nossos pacientes. O seguimento rigoroso com a vigilância e tratamento dos fatores de risco para as doenças cardiovasculares podem melhorar ainda mais os resultados a longo prazo da cirurgia do AAA em pacientes jovens.

\section{Referências}

1. Blanchard JF. Epidemiology of abdominal aortic aneurysms. Epidemiol Rev. 1999;21:207-21.

2. Pleumeekers HJ, Hoes AW, van der Does E, et al. Aneurysms of the abdominal aorta in older adults: the Rotterdam study. Am J Epidemiol. 1995;142:1291-9.

3. Muluk SC, Gertler JP, Brewster DC, et al. Presentation and patterns of aortic aneurysms in young patients. J Vasc Surg. 1994;20:880-6; discussion 887-8.
4. Cherr GS, Edwards MS, Craven TE, et al. Survival of young patients after abdominal aortic aneurysm repair. J Vasc Surg. 2002;35:94-9.

5. Copeland GP, Jones D, Walters M. POSSUM: a scoring system for surgical audit. Br J Surg. 1991;78:355-60.

6. Mingoli A, Sapienza P, Feldhaus RJ, di Marzo L, Burchi C, Cavallaro A. Aortoiliofemoral bypass graft in young adults: long-term results in a series of sixty-eight patients. Surgery. 1997;121:646-53.

7. Valentine RJ, Grayburn PA, Eichhorn EJ, Myers SI, Clagett GP. Coronary artery disease is highly prevalent among patients with premature peripheral vascular disease. J Vasc Surg. 1994;19:668-74.

8. Martin GH, Allen RC, Noel BL, et al. Carotid endarterectomy in patients less than 50 years old. J Vasc Surg. 1997;26:447-54; discussion 454-5.

9. Bonamigo TP, Lucas ML, Erling Jr N. Tratamento cirúrgico dos aneurismas da aorta abdominal: existe diferença nos resultados entre homens e mulheres? J Vasc Bras. 2006;5(2):101-8.

10. Harris LM, Peer R, Curl GR, Pillai L, Upson J, Ricotta JJ. Long-term follow-up of patients with early atherosclerosis. J Vasc Surg. 1996;23:576-80; discussion 581.

Correspondência:

Dr. Telmo P. Bonamigo

Rua Coronel Bordini, 675/304

CEP 90440-001 - Porto Alegre, RS

E-mail: telmobonamigo@terra.com.br 